REGARDS

SUR L'ECONOMIE ALLEMANDE

BULLETIN ECONOMIQUE DU CIRAC

\section{Regards sur l'économie allemande}

Bulletin économique du CIRAC

$113 \mid 2014$

Varia

\title{
Les élections au Parlement européen
}

État de l'euroscepticisme en France, en Allemagne et en Europe. Quelles conséquences pour la poursuite de l'union politique européenne?

Roland Cayrol, Christian Lequesne, Thomas Petersen, Henrik Uterwedde et Isabelle Bourgeois

\section{(2) OpenEdition}

\section{Journals}

Édition électronique

URL : http://journals.openedition.org/rea/4681

DOI : $10.4000 /$ rea.4681

ISSN : 1965-0787

Éditeur

CIRAC

Édition imprimée

Date de publication : 15 juillet 2014

Pagination : 5-16

ISSN : 1156-8992

\section{Référence électronique}

Roland Cayrol, Christian Lequesne, Thomas Petersen, Henrik Uterwedde et Isabelle Bourgeois, « Les élections au Parlement européen », Regards sur l'économie allemande [En ligne], 113 | juillet 2014, mis en ligne le 17 juillet 2016, consulté le 02 mai 2019. URL : http://journals.openedition.org/rea/4681

DOI : 10.4000/rea.4681 


\section{Les élections au PE.}

Etat de l'euroscepticisme en France, en Allemagne et en Europe. Quelles conséquences pour la poursuite de l'union politique européenne?

\author{
R. Cayrol, C. Lequesne, T. Petersen, H. Uterwedde \\ Débat animé par I. Bourgeois
}

\begin{abstract}
Isabelle BOURGEOIs : Les élections européennes qui se sont achevées le 25 mai soulèvent un certain nombre de questions. Nous avions à élire notre parlement de citoyens européens. Mais qu'est-ce qu'un citoyen européen? Nous ne le savons pas. Quelle est la fonction de ce Parlement européen? Elle ne s'impose pas à l'évidence. Nos députés à Strasbourg et Bruxelles, nous ne les connaissons guère non plus, à part quelques têtes de liste que nous avons découvertes peu avant le scrutin. Quant à l'adjectif européen, il est lourdement surchargé de sens les plus divers et les plus contradictoires : paix, prospérité, modèle social, mais aussi cause de toutes les crises qui nous frappent, source de tous les maux : chômage, précarisation, euro fort, perte de souveraineté, immigration incontrôlée, dilution de nos valeurs, déficit démocratique, etc.
\end{abstract}

Qu'est-ce que l'Europe ? La question est déterminante pour notre adhésion à ce projet que nous poursuivons en commun depuis plus de 60 ans. Mais il est impossible d'y répondre simplement. Les réalités institutionnelles sont complexes, le changement que nous vivons nous fait peur. L'Europe s'est élargie à 28 Etats membres, elle a une monnaie en vigueur dans 18 Etats déjà ; mais le marché intérieur est loin d'être parachevé. La mobilité des personnes et des biens est largement assurée, celle des capitaux a progressé ; maintenant se pose la difficile question de l'articulation entre nos modèles d'Etat-providence. L'Union a quelques institutions communautaires ; mais leur articulation reste à définir plus précisément, entre elles, entre l'échelon communautaire et l'échelon national - et même l'échelon régional.

L'Europe politique reste à construire. Voulons-nous une fédération? Si oui, ce qui est loin de faire consensus, alors comment doivent s'articuler les compétences respectives des Etats membres et de l'UE ? Quels rouages définir exactement pour la "démocratie représentative" dans l'UE ? Nous nous trouvons aujourd'hui à une croisée des chemins. Et c'est peut-être la difficulté à affronter ce défi décisionnel qui explique le résultat du scrutin de ces élections européennes. Ne serait-ce pas notre profonde peur de décider que révèle la montée de l'euroscepticisme, du souverainisme et des populismes de tous bords?

\section{Un vote débouchant sur une 'grande coalition'}

Christian LEQUESNE : La première chose que nous remarquons à l'échelle des Vingt-Huit est un taux de participation qui se maintient par rapport à la dernière élection en 2009, sans que nous ayons d'ailleurs vraiment d'éléments d'explication. Le soir du scrutin, nombre d'hommes et de femmes politiques ont présenté cela comme le résultat de la «politisation » de l'enjeu : le fait qu'on vote pour un Parlement qui devrait, si ceci intervient, élire le président de la Commission. Je pense qu'une l'hypothèse plus plausible est qu'il existe une petite montée

\section{Synthèse}

du débat

organisé le

02-06-2014

à Paris

par le CIRAC

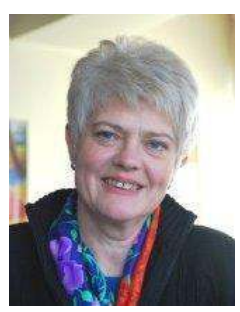

Isabelle Bourgeois, rédactrice en chef de Regards sur l'économie allemande 


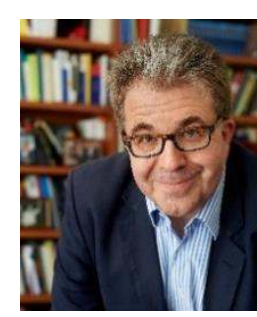

Christian Lequesne, directeur de recherche au CERI et professeur à Sciences $\mathbf{P O}$

L'équilibre au centre du PE est préservé.. ...malgré la montée des extrêmes

Renforcement des votes de grande coalition au PE ?

Comment vont s'impliquer les députés d'extrême-droite au PE ? des extrêmes, qu'il ne faut toutefois pas surestimer, et que, par définition, les extrêmes se mobilisent toujours beaucoup au moment du vote.

Nous sommes, en France, très polarisés sur le résultat du FN qui arrive en première place avec 24 élus sur 74. Mais l'équilibre du Parlement européen reste le même qu'en 2009 : les deux principaux groupes - le centre droit (le Parti populaire européen, PPE) et les sociaux-démocrates de l'Alliance progressiste des Socialistes et Démocrates (S\&D) - continuent à avoir la majorité absolue. En outre, les Libéraux restent le troisième groupe et Les Verts le quatrième. Les extrêmes n'ont pas la majorité au sein du Parlement européen. Toutefois, cela pose un problème particulier à la France car, dans ce Parlement, il est important d'avoir un maximum de députés au sein des deux grands groupes. Or c'est précisément ce que la France n'aura pas. Cela constitue à l'inverse un avantage pour d'autres pays comme l'Allemagne, puisque la Christlich Demokratische Union (CDU) a fait un très beau score et sera bien représentée au sein du groupe PPE. Je pense aussi aux Italiens du Parti démocrate qui seront très représentés au sein du groupe S\&D.

Pour ce qui est de la montée des extrêmes, il est vrai que, notamment à droite, mais pas seulement si on considère la Grèce, ils ont tendance à gagner des voix. Il est cependant assez difficile pour l'instant de déceler un paradigme commun qui permettrait de mettre, sous un même schéma explicatif, l'ensemble des extrêmes. Les variations nationales sont très importantes. Néanmoins, on peut constater que tous ces partis sont eurosceptiques, voire europhobes. Tous ont un discours de rejet de l'establishment, c'est-à-dire des partis traditionnels, à l'instar de Nigel Farage à la tête du parti United Kingdom Independance Party (UKIP) au Royaume-Uni. Tous ces partis sont plutôt protectionnistes en termes économiques. Et tous ces partis ont un discours de défense de l'Etat social.

Dans quelle mesure cela pèsera-t-il sur les votes ? C'est très difficile à dire car il n'y a pas de structuration des votes au sein du PE. Des études sur la dernière législature montrent que dans la période 2009-2014, $30 \%$ des votes seulement ont été motivés par la logique gauche-droite que nous connaissons dans beaucoup de pays de l'UE, $30 \%$ par une logique de grande coalition - c'est-à-dire le S\&D et le PPE ensemble -, les $40 \%$ restants étant des votes pris par consensus à géométrie variable. Toute la question sera de savoir si les votes de grande coalition vont se renforcer avec la présence accrue des extrêmes.

Si parfois, dans la logique gauche-droite, on tolèrera que l'extrême-gauche et l'extrême-droite se joignent soit aux Verts et aux Socialistes pour la gauche, soit au PPE et aux Libéraux pour la droite, cela n'a rien d'évident. Pour les $40 \%$, cela posera la question de l'implication des députés de ces groupes dans le travail parlementaire concret. Or on constate que lors de la législature précédente, le $\mathrm{FN}$ - pour prendre l'exemple français - s'y est très peu impliqué. La question est de savoir si cela va continuer ou si, au contraire, il va y avoir, dans la lignée de ce qu'on observe dans le discours du FN en France, la quête de l'image d'un parti normal. On doit se poser la question aussi pour les autres partis, sachant qu'actuellement, les grandes manœuvres ont lieu pour essayer de former des groupes parlementaires. Sur ce point, le Front national n'a pas de difficulté avec le critère du nombre de membres puisqu'il en faut 25 pour former un groupe, mais ceux-ci doivent être originaires de sept pays. Or cela est délicat du fait que certains partis de la droite extrême, notamment le parti populaire danois et le parti finlandais, n'ont pas fait un très bon score. Quant au UKIP de Nigel Farage, il a fait savoir qu'il n'était pas question de faire alliance avec le FN en raison des déclarations sulfureuses de Jean-Marie Le Pen, le père de l'actuelle leader, sur la question des chambres à gaz, qui disqualifient le FN y compris chez certains partis de la droite extrême.

Pour le gouvernement français qui sera obligé de travailler avec le Parlement européen, la situation ne sera pas simple : il ne faut pas oublier qu'aujourd'hui, environ $70 \%$ des questions sont co-décidées entre le Parlement européen et le 
Conseil des ministres, c'est à dire des 28 Etats. Ce sera plus simple pour Angela Merkel, surtout dans une situation de grande coalition, car le gouvernement français ne pourra pas compter sur l'appui d'un nombre de représentants important dans les deux grands groupes parlementaires européens.

\section{France et Allemagne : une large adhésion à l'Europe}

Roland CAYROL : Sur la France, on dit beaucoup de choses très simplificatrices et souvent erronées. Jusqu'aux années 1970, on pouvait facilement isoler dans l'opinion française des gens qui étaient pour l'Europe et des gens qui étaient contre. On pouvait dire que les électeurs se réclamant du gaullisme et du communisme - qui à l'époque pesaient beaucoup - étaient hostiles à l'intégration européenne et que tous les autres y étaient favorables. Nous avions donc, seIon les moments, une grosse et une petite moitié de Français qui s'opposaient sur la question européenne. Cette période est révolue. Les enquêtes qualitatives en profondeur nous montrent que, désormais, presque chaque citoyen français est un composé d'adhésion à l'Europe et de réticence ou de crainte à son égard. II n'y a plus d'opposition entre des Français pro-européens (qui avaient à un moment mis la France en tête des pays les plus européens de l'Eurobaromètre) et des anti-européens. Tout le monde est partagé. L'énorme majorité des Français dit être favorable à l'Europe car c'est bon pour la France, pour l'avenir, pour leurs enfants et que, d'une certaine façon, c'est naturel. Comme le disait François Mitterrand dans une phrase qui serait adoptée par la majorité des Français: "L'Europe est l'avenir de la France. " Tout cela est accepté très massivement dans l'opinion publique, pratiquement tous courants confondus. Le substrat pro-européen de ce pays est largement dominant.

Mais au fil du temps est née aussi une réticence ou une crainte par rapport à l'Europe sur deux thèmes. Celui de notre fameux modèle social : nous sommes très fiers d'avoir le "plus beau modèle social du monde ", même quand c'est faux. Nous ne voulons donc surtout pas que les autres - et notamment Bruxelles - nous empêchent de bénéficier de ce modèle. II existe donc une crainte que l'accélération de l'Europe fasse un nivellement sur le modèle des moins chanceux - c'est alors souvent les Portugais qu'on cite dans les enquêtes qualitatives - et que nous y perdions. La deuxième cause de réticence est notre mode de vie : les Français sont persuadés que la France (et chaque membre d'une région pense que sa région et sa commune plus encore) est l'endroit où il fait le meilleur vivre au monde. La crainte de l'Europe - qui est la même que la crainte de la mondialisation -, c'est la crainte de l'uniformité technocratique des normes qui vont petit à petit nous priver de notre art de vivre, c'est-à-dire en premier lieu notre gastronomie. Un jour, la Commission européenne, dans un bon mouvement, avait décidé qu'on pouvait faire une exception à l'utilisation du lait pasteurisé pour les produits laitiers et accepter le lait cru dans les régions de production où c'était une tradition culturelle. L'opinion française a immédiatement compris que les technocrates bruxellois voulaient nous empêcher de faire du camembert au lait cru. Cette histoire, qui a plus de 20 ans, est restée incroyablement vive dans les mémoires.

Cette double réticence a pris corps progressivement. Elle a été rejointe plus récemment par une raison qui touche à la crise et qui est plus profondément politique. La France est en crise : les gens sont taraudés par le chômage croissant, ils sont inquiets pour le pouvoir d'achat et ils ont peur pour l'avenir de leurs enfants. Or dans cette situation, on s'en prend aux décideurs - notre gouvernement, notre président de la République et l'Europe - qui font que malgré toutes les promesses politiques, nous ne sortons pas de la crise. On sait bien que l'Europe prend désormais de nombreuses décisions. Par conséquent, l'Europe ne réussit pas mieux que notre gouvernement et notre Parlement. II est donc normal qu'on en veuille autant aux autorités européennes qu'on en veut

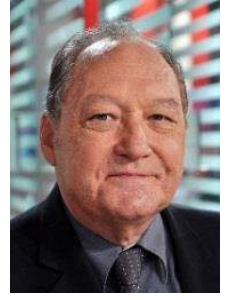

Roland Cayrol, directeur de recherche associé au CEVIPOF et fondateur de l'institut CSA

\section{Les Français}

sont partagés sur l'Europe

Les peurs françaises font l'amalgame entre Europe et mondialisation

Crise générale de confiance dans les institutions 


\section{Mais le substrat pro-européen domine}

Après la guerre, seule l'Europe offre une perspective aux Allemands

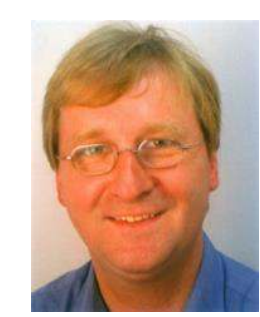

Thomas Petersen, chercheur à l'Institut für Demoskopie d'Allensbach

Aujourd'hui : lassitude épidermique, ...

... mais les Allemands son restés foncièrement européens aux autorités françaises. Je rappelle que le président de la République est au plus bas dans l'histoire de nos sondages. Les uns et les autres sont pris dans ce même mouvement de critique et de scepticisme.

Mais on ne peut pas en conclure qu'un courant d'opinion eurosceptique est en train de se créer. II s'agit plutôt d'un mélange extrêmement complexe d'adhésion profonde à l'unité européenne et de scepticisme. Bien souvent, quand on demande aux Français s'il vaut mieux régler au niveau national ou européen les problèmes ayant trait au chômage, à l'environnement ou à la sécurité, ils répondent à une écrasante majorité qu'il serait plus efficace de les résoudre au niveau européen, y compris quand ces personnes figurent parmi les eurosceptiques. Je rappelle par ailleurs que plus des deux tiers des Français n'entendent pas qu'on quitte jamais l'UE et que les trois quarts ne veulent pas du tout quitter l'Euro. Au-delà de l'analyse des résultats électoraux, on observe ainsi, dans les enquêtes d'opinion dont nous disposons, que l'euroscepticisme n'a pas envahi les consciences de ce pays. C'est plus complexe que cela.

Thomas Petersen : La plupart de ce qui vient d'être dit est applicable à l'Allemagne, avec certaines nuances certes, et dans un contexte différent : nous ne traversons pas la crise structurelle et économique que connaît la France, mais nous l'avons subie il y a dix ans. Pour comprendre l'ambiguïté du rapport à l'Europe qu'ont les Allemands, il faut revenir sur l'histoire. Le tout premier sondage de I'Institut für Demoskopie d'Allensbach a été mené en 1947, dans la zone d'occupation française, auprès d'écoliers et d'étudiants. La famine régnait, les Allemands étaient désorientés. On leur a demandé : "Vous intéressez-vous à la politique, aux partis nouvellement fondés ? ». Le processus de création de la République fédérale d'Allemagne se mettait alors en place, mais une immense majorité a répondu qu'elle ne voulait plus jamais avoir affaire avec la politique. L'enquête comportait ensuite quelques questions sur ce qu'on nommait alors les Etats-Unis d'Europe. Les réponses apparurent comme un rayon de soleil dans toutes les réponses teintées de déprime. En 1947, après des guerres et des dictatures dévastatrices et après un siècle marqué par de violents et nombreux conflits entre Européens, les Allemands estimaient qu'une organisation commune au continent serait peut-être la seule perspective d'avenir. Cette tendance se poursuit dans les années 1950 et 1960, où ce rêve commence à se concrétiser avec une rapidité que les deux générations précédentes n'auraient pu imaginer. Or à partir de la fin des années 1960, on constate que plus ce rêve se concrétise, plus les gens s'aperçoivent que l'Europe ne constitue pas la panacée et qu'elle devait être organisée. II y avait des malentendus à résoudre et des compromis à négocier, avec les Anglais, les Français. Les problèmes et les querelles font partie du processus.

Ainsi, une certaine lassitude est venue altérer le caractère globalement europhile des Allemands exprimé pendant toutes ces années. Lorsqu'on les questionne sur l'Europe aujourd'hui, ils s'empressent d'exprimer leur mécontentement envers la bureaucratie de Bruxelles et de se plaindre de l'ingérence de l'Europe dans tous les domaines. Au cas du camembert évoqué par Roland Cayrol correspond en Allemagne celui de la loi allemande sur la pureté de la bière que les Européens ont voulu interdire. Or les Allemands de leur côté exigent que la bière de moins bonne qualité ne puisse pas arriver sur leur territoire. II s'agit ici d'une querelle superficielle typique qui apparaît partout dès que les choses commencent à devenir sérieuses.

Mais ce mécontentement n'apparaît qu'en surface. Car la sympathie des Allemands pour l'Europe est restée constante. Même dans les moments critiques, la grande, voire l'écrasante majorité des Allemands reste disposée à payer pour l'intégration européenne, et ce malgré le mécontentement face à la bureaucratie, au manque de consensus et à la situation difficile de l'économie européenne. Les Allemands déplorent également que les pays de l'Europe du Sud comme l'Italie ou la Grèce, qui ont intégré l'Euro sur la base de statistiques erronées, tendent la main en demandant aux Allemands de payer maintenant que 
l'argent facile s'est fait rare. II n'est pas simple pour les responsables politiques de convaincre les citoyens qu'ils se doivent pourtant de le faire. Cela explique la présence de partis euro-critiques en Allemagne, bien qu'il semble étonnant qu'ils n'aient pas pris plus d'importance. Cela dit, sous les tracas du quotidien demeure la conviction inébranlable que cette Europe commune en vaut le prix.

\section{Eurosceptiques, nationalistes, souverainistes : qui sont-ils ?}

T. PETERSEN : L'Alternative für Deutschland (AfD), ce nouveau parti créé il y a un peu plus d'un an, n'a rassemblé que $7 \%$ des voix. Contrairement à la France, l'Allemagne n'a pas connu de séisme politique, mais plutôt une migration de voix. L'AfD n'est pas le FN, il ne se situe pas à l'extrême-droite du spectre politique. C'est au contraire un parti qui, bien que conservateur en ce qui concerne les valeurs de la société, est très libéral en matière économique : il a été créé et est dirigé par des économistes. Les opinions qu'il représente s'inscrivent toutes dans le camp libéral-conservateur, de même que ses membres et ses électeurs se recrutent parmi les adhérents des partis de l'Union (CDU et CSU) ; le président de l'AfD fut longtemps membre de la CDU. La seule raison d'être de ce parti est de rassembler ceux qui, en Allemagne, sont contre l'Euro - pas tant contre I'UE que contre la monnaie unique. $\mathrm{Si}$, pour une raison ou une autre, l'AfD obtenait la majorité absolue lors d'élections anticipées au Bundestag, il n'y aurait pas à s'inquiéter pour la démocratie ni pour les droits de l'Homme, et pas non plus pour l'Union européenne. Et l'Euro ne serait pas en danger non plus, puisqu'il est impossible de faire machine arrière.

Par contre, la montée de l'AfD révèle une tendance bien plus inquiétante à un autre niveau. Ce parti est nationaliste, au sens originel du terme : il n'est pas d'extrême-droite, c'est-à-dire ni militariste ni agressif, mais ses positions sont toutes centrées exclusivement sur la Nation et la question de savoir ce qui est immédiatement profitable à l'Allemagne, l'AfD rejetant tout ce qui ne l'est pas. II y a en un sens une certaine normalité dans l'émergence de ce parti : il exprime aussi une tendance, répandue surtout dans les jeunes générations, de ne plus avoir honte du drapeau allemand. Le traumatisme du III Reich, qui marquait les générations précédentes, et qui était aussi, il faut le rappeler, une des forces motrices de l'intégration européenne, tend à s'effacer. Défendre les intérêts de l'Allemagne est devenu aussi normal que défendre les intérêts de la France. Ce constat n'enlève rien au fait que, en Allemagne comme dans l'UE, ce parti hostile à l'Euro pose problème.

Henrik UTERWEDDE : Si on ajoute aux $7 \%$ de l'AfD le $1 \%$ de voix des néo-nazis du Nationaldemokratische Partei Deutschlands (NPD), cela fait tout juste $8 \%$. En France, si on additionne le FN, le Front de gauche et les quelques souverainistes qui partagent la même haine contre le soi-disant libéralisme, contre l'Europe et contre le principe de responsabilité d'un parti de gouvernement, on arrive à environ $36 \%$. Thomas Petersen a bien expliqué la nature très différente des eurosceptiques allemands et français. Par exemple, à une semaine du scrutin, le philosophe Michel Onfray consacrait deux pages aux élections européennes dans le Nouvel Observateur et y dénonçait à plusieurs reprises le libéralisme et l'Europe libérale. On peut se demander quel est le degré de libéralisme de l'UE. Est-ce que les règles budgétaires sont du libéralisme ? Ce genre de débat n'existe pas en Allemagne.

Plusieurs facteurs entrent en jeu pour expliquer le vote. En France, il s'agit de prendre position par rapport à l'Europe ou de sanctionner le président de la République et l'austérité. En Allemagne à l'inverse, l'économie se porte bien, le taux de chômage est d'environ $5 \%$, et la grande coalition vient de dépenser beaucoup d'argent sur le volet social, sans penser à l'avenir du pays. Pourquoi les Allemands sanctionneraient-ils Angela Merkel ? Par ailleurs, les craintes des Allemands vis-à-vis de l'euro sont diffuses, sauf celles, qui ont toujours existé, sur le danger que les citoyens allemands aient à payer pour tous les «cancres »
L'AfD :

un parti libéral-conservateur rassemblant les déçus de l'Euro

L'AfD

est révélateur de

la montée des nationalismes

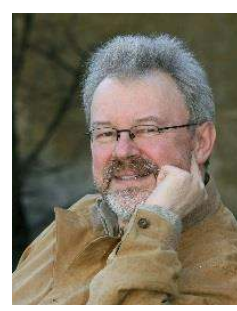

Henrik Uterwedde, directeur adjoint de 1'Institut Franco-allemand (DFI), Ludwigsburg

Les électeurs allemands ne cherchaient pas à sanctionner la chancelière 
La présence d'un député NPD au PE est purement arithmétique

De grandes différences entre FN et Front de gauche

Plus important que l'opposition gauche-droite, celle entre libéralisme et autoritarisme

En France, connotation négative du libéralisme

Nos omissions sur l'Europe ont fait le lit du nationalisme de l'Europe réunis. Le vote allemand fait apparaître un élément très intéressant. Le parti bavarois, la Christlich-Soziale Union (CSU), a cru bon de se distinguer de la CDU. II a placé en tête de liste Peter Gauweiler, un eurosceptique avéré. Dès lors, tandis que la CDU d'Angela Merkel a gagné en pourcentage de votes, la CSU bavaroise a chuté de 10 points, comme si les électeurs exprimaient le fait que quitte à être contre l'Euro et à être eurosceptique, mieux valait voter pour l'original, et donc l'AfD.

T. Petersen : Il faut évoquer aussi le rôle que joue dans la montée des extrêmes une modification récente du mode de scrutin allemand. Normalement, un parti doit obtenir $5 \%$ des voix pour entrer au Bundestag ou dans un parlement régional. Cette disposition est un enseignement tiré de l'instabilité de la République de Weimar dont la multiplication des mini-partis plus radicaux les uns que les autres avait scellé la fin. Or le Tribunal constitutionnel fédéral vient de supprimer ce seuil pour les élections européennes, estimant qu'il n'est pas démocratique puisqu'il interdit de fait aux électeurs des petits partis de participer à la démocratie dans l'Union. C'est la raison pour laquelle, pour la première fois, se trouvent représentés au Parlement européen des formations aussi peu conventionnelles que celle défendant la protection des animaux ou la fidélité à la Bible - et aussi le parti NPD. Que ce dernier ait un député européen est la conséquence arithmétique du fait qu'il suffit désormais, étant donné la taille de l'Allemagne, de moins de $1 \%$ des voix pour avoir un siège au $P E$.

R. CAYrol : J'estime pour ma part qu'il n'est pas légitime 'politologiquement' et qu'il n'est jamais bon pour comprendre le spectre politique d'additionner la gauche de la gauche et l'extrême-droite. En effet, tout les oppose. Sur l'Europe, le Front de gauche ne demande pas de quitter l'UE ni l'Euro. II s'en prend au libéralisme, incarné par Bruxelles mais aussi par notre gouvernement. C'est une position sur l'orientation donnée aux politiques. Je ne classerais donc pas le Front de gauche - tous courants confondus - parmi les anti-européens.

T. Petersen: Je pense que, bien au contraire, il faut mettre dans la même catégorie les extrêmes de droite et de gauche, même s'ils se distinguent sur de nombreux points. Car à l'échelle gauche-droite du spectre politique s'en ajoute une autre : celle où s'opposent libéralisme et autoritarisme. Et c'est cela que partagent les extrêmes de gauche comme de droite : ils mènent tous une lutte contre l'Etat libéral et contre l'Europe.

R. CAYrol: Certes, c'est une lecture possible, mais le faire pour l'Europe me semble une erreur. En France, leurs positions sur l'Europe ne sont absolument pas les mêmes. Le Front de gauche n'est pas pour la sortie de l'Europe ni de l'Euro alors que le FN et les souverainistes comme Nicolas Dupont-Aignan sont pour la sortie de l'Union européenne. II s'agit là d'une différence majeure.

C. LEQUESNE : Je pense moi aussi qu'il faut les distinguer. Le rapport à l'Europe est tout aussi négatif à l'extrême-gauche qu'à l'extrême-droite, mais pour des raisons complètement différentes. Dans le fond, l'extrême-gauche est plus représentative d'un problème très français qui est le rapport au libéralisme. II est lié à l'histoire française et au fait que la pensée libérale a été faible dans le développement de la pensée politique française. En effet, l'État était très puissant. Le libéralisme reste aujourd'hui complètement inscrit dans le sens commun comme quelque chose de négatif.

\section{Un déni d'Europe dans les espaces publics}

T. Petersen : La naissance de l'AfD et sa montée dans l'opinion sont aussi la conséquence d'une gigantesque erreur que nous avons commise en Europe au cours des vingt ou trente dernières années : celle de toujours justifier la progression de l'intégration européenne par la raison économique. Nous n'avons cessé d'affirmer que l'Euro nous apporterait croissance et emplois. Tant mieux s'il en est ainsi. Mais ce n'était pas la vraie raison de la création de la monnaie 
unique. Donc quand on ne cesse d'avancer une justification économique et que, soudain, l'Europe se met à traverser une crise économique, il est naturel de penser que l'Euro était un mauvais choix puisqu'il n'a pas tenu ses promesses. La montée de l'AfD est la réponse au fait que nous avons omis de dire pourquoi nous construisons l'Europe ; vue sous cet angle, elle n'a pas de quoi nous inquiéter outre mesure.

H. UTERWEDDE : Je pense qu'il y a un manque de repères sur l'Europe, pas seulement en France, mais dans de nombreux pays européens, y compris en Allemagne. Comment voulez-vous que les gens votent pour des partis 'responsables' et se mobilisent pour l'Europe si les hommes politiques et les grands partis ne font pas montre de foi en l'Europe ? " L'offre politique en France, mais aussi dans les autres pays, est tellement médiocre vis-à-vis des électeurs. Comment voulez-vous que les gens se mobilisent pour aller voter pour l'Europe? ", a dit récemment l'ancien député européen Jean-Louis Bourlanges. Nos gouvernements n'ont plus la foi et ne sont plus capables d'expliquer l'Europe. Helmut Kohl disait que pour lui, l'Euro était une question de guerre et de paix. Il a dit à tous ceux qui étaient contre l'Euro, les banquiers et les milieux financiers en Allemagne, que la monnaie européenne était trop importante pour la laisser aux seuls spécialistes de l'économie et des finances. Et il avait raison. Mais en France, cette foi européenne s'est perdue depuis très longtemps. En Allemagne, Gerhard Schroeder et Angela Merkel ne sont pas des Européens patentés. J'émettrais donc quelques réserves quant à la participation stable et au résultat assez rassurant en Allemagne. L'Allemagne ne traverse certes pas les problèmes politiques de la France, mais les problèmes des autres sont aussi les nôtres en Europe. Est-ce que le fait de dire qu'au fond, on est pour l'Europe, suffira à l'avenir quand les décisions à prendre auront un impact plus net sur le mode de vie et sur le cœur de la souveraineté ?

R. CAYrol: Durant la campagne électorale, sur Internet, de nombreux journalistes ou dirigeants politiques de la CDU ou du parti social-démocrate (Sozialdemokratische Partei Deutschlands, SPD) venaient expliquer et défendre l'Union européenne. Or cela n'a pas du tout existé en France. Les deux grands partis gouvernementaux, I'UMP et le PS, n'ont cessé de critiquer les manques de l'Europe. Nous avons pris l'habitude d'avoir des gouvernants qui considèrent Bruxelles comme le 29e Etat de l'Union qui impose sa loi à tous les autres. Nous avons pris l'habitude d'avoir un spectre gouvernemental centre-gauche centre-droit qui n'est que critique à l'égard de l'Europe. Si dans leurs textes fondamentaux, ces partis sont pour l'Union européenne, ils ne la défendent pas et ne la valorisent pas. Cette campagne était de ce point de vue absolument lamentable. Deux petits partis ont progressé : les centristes et Les Verts, qui sont précisément les deux seuls à avoir fait campagne pour l'Europe et pris des voix aux grands partis qui n'osaient pas avouer qu'ils étaient pro-européens. L'offre politique montre que la situation est bien pire en France qu'en Allemagne.

T. Petersen : En Allemagne aussi, les partis démocratiques manquent de vigueur à promouvoir l'Europe. C'est aussi une question de génération. J'ai toujours en tête cette phrase de Wolfgang Schüssel, l'ancien chancelier autrichien, qui disait que si vous voulez comprendre l'enjeu européen, alors il faut aller regarder les monuments aux morts dans les cimetières des villages. Le problème, c'est que la génération qui a vécu les guerres s'éteint progressivement, et que ces guerres ne sont qu'un souvenir lointain pour les générations suivantes. Si nous voulons que l'idée d'Europe reste vivante, il faut donc la promouvoir activement. Je crois que nous commettons à propos de l'Europe la même erreur qu'en Allemagne après l'Unité : nous étions alors convaincus que le progrès qu'apportent la démocratie, la liberté et l'Etat de droit est si évident qu'il n'est pas besoin de l'expliquer. Au contraire, il faut inlassablement dire ce que nous apporte l'Europe. Je me souviens d'un incident révélateur. Peu avant les élections européennes de 2009, dans le cadre d'une conférence sur la révision du texte du projet de traité constitutionnel rejeté par la France et les Pays-Bas, un député
Partout, il y a un manque de repères sur le projet européen

En France, aucun parti n'ose défendre l'Europe

L'Europe manque cruellement de symboles 
Comment légitimer l'Europe à partir des valeurs ?

Les valeurs semblent acquises aux yeux de l'opinion

La crise en Ukraine vient rappeler que l'Europe est œuvre de paix

La question aujourd'hui est : quelle Europe voulons-nous?

\section{Des compromis sont nécessaires pour faire avancer l'Europe}

européen du PPE avait glissé : "Nous avons pu nous débarrasser de toutes ces choses inutiles comme l'hymne et le drapeau ». Croire que des symboles ne sont pas importants est une grave erreur d'appréciation; ce sont eux au contraire qui permettent de s'identifier avec l'Europe.

C. LEQUESNE : L'absence de grand pédagogue aujourd'hui au PS ou à I'UMP en France est liée au fait qu'ils sont divisés sur la question européenne à l'intérieur. Si on est plutôt de centre-droit, mieux vaut 'vendre' l'Europe au sein de I'Union des démocrates indépendants (UDI) et du Mouvement démocrate (Modem). Quant au PS, il reste profondément divisé par les résultats du référendum de 2005. Plus généralement, il devient beaucoup plus difficile, dans tous les pays de l'UE, de légitimer l'Europe à partir des valeurs dans un discours politique crédible. Mes collègues tchèques, polonais et slovaques me disent que les hommes politiques de leur pays ne peuvent plus évoquer les valeurs. Aujourd'hui, il faut légitimer l'Europe par l'utilitarisme et par l'économie : qu'estce que cela nous a apporté, quid de l'égalisation des salaires... ? II n'en demeure pas moins que d'un point de vue normatif, la légitimation par les valeurs serait nécessaire. Toutefois, d'un point de vue électoral, je ne suis pas sûr que ce soit une façon de recueillir l'adhésion des électeurs.

R. Cayrol : Le discours sur les valeurs fuit progressivement toute la politique, pas seulement sur l'Europe. Dans chacun de nos pays, il diminue du fait de la crise bien sûr, mais peut-être aussi parce que toutes ces valeurs fortes et fédératrices de paix et de démocratie qui ont justifié les avancées européennes sont devenues molles. Aux yeux de l'opinion, elles n'ont plus besoin d'être défendues dans la mesure où elles sont acquises et semblent aller de soi.

T. Petersen : Je suis moins pessimiste face à cette évolution assez récente. En effet, personne, au plus tard depuis le départ de Helmut Kohl, n'a plus réellement tenté ce discours de valeurs. Or aujourd'hui, la crise en Ukraine change la donne. Quand on demande aux Allemands de dire ce qu'est pour eux l'Europe, ce qui est le plus important à leurs yeux, ils répondent beaucoup plus souvent que ces dernières années encore qu'elle œuvre pour la paix. Autrement dit, tant que toutes ces valeurs ne semblent pas menacées, on les perd de vue. Quand François Mitterrand, dans son dernier grand discours devant le Parlement européen, assénait : "Le nationalisme, c'est la guerre », il ne récolta que railleries. Cette phrase est pourtant plus vraie que jamais. Et j'aimerais entendre plus souvent les responsables politiques allemands en prononcer de semblables.

H. Uterwedde : Vladimir Poutine semble nous rappeler à point nommé que nous avons besoin de la volonté de partager des valeurs, mais aussi des intérêts fondamentaux, et que, isolés, nous ne sommes rien ou presque.

La question que nous devons nous poser aujourd'hui, c'est: Quelle Europe voulons-nous? II y a de la place pour des arguments socialistes, keynésiens, conservateurs et libéraux. II est nécessaire et légitime que s'opposent plusieurs visions politiques de l'économie et de la société, cela fait partie de la démocratie. Les élections au Parlement européen devraient être l'occasion de mener de tels débats - mais sans perdre de vue ce qui est essentiel. Nous appartenons à une communauté de destin. II n'y a pas qu'une seule voie possible. Dans cette dialectique, l'avantage psychologique est peut-être aux Allemands parce que ce sont les députés allemands de centre-gauche et centre-droit qui vont décider au PE, composé majoritairement des sociaux-démocrates du PSE et de députés du PPE. Ce sont eux qui vont trouver - je l'espère - les compromis nécessaires pour faire avancer l'Europe.

\section{Quelle démocratie pour l'Europe?}

Au niveau des institutions, l'Europe repose sur le compromis. Au Parlement européen, elle s'apparente à une grande coalition permanente entre les deux forces politiques PSE et PPE. En outre, au Conseil, il est nécessaire de trouver 
des majorités parmi les 28 gouvernements représentés. Or cette situation de grande coalition est connue dans la vie politique allemande. Les quatre grands partis n'ont aucun état d'âme à travailler ensemble quand l'électeur l'a voulu. Alors qu'en France, on retombe vite dans le manichéisme. Je souhaite que la culture politique française puisse progressivement évoluer dans un sens qui valorise davantage la recherche de compromis. Sans cet esprit constructif, nous ne serons pas capables d'affronter des controverses politiques au niveau européen.

R. CAYRoL : Nous sommes dans une Union où la majorité des pays pratique la représentation proportionnelle pour les élections parlementaires. Les gouvernements procèdent de la représentation proportionnelle et du Parlement. Cela engendre ainsi des alliances et, partant, cette nécessité de compromis. D'une certaine façon, les habitants de ces pays regardent le PE comme une institution qui ressemble au Parlement national : des endroits où on parlemente, et où il faut se mettre d'accord. Ce n'est pas le cas pour les Britanniques et les Français. En ce qui nous concerne, les élections européennes sont les seules où nous votons à la représentation proportionnelle. C'est pourquoi, depuis les premières élections au PE au suffrage universel direct en 1979, tous les commentaires des journalistes et des hommes politiques le soir du scrutin se révèlent faux. Ils disent à chaque fois que les lignes politiques habituelles ont été absolument effacées et que rien ne sera plus comme avant. Or c'est à chaque fois faux. Nous revenons toujours à la logique du système majoritaire en France. Par conséquent, nous verrons que ce sera tout aussi faux cette fois-ci.

En France, il est très étrange de voir une campagne électorale pour les élections européennes se jouer sur un scénario gauche-droite, alors que le citoyen de base, qui ne sait déjà pas à quoi sert le Parlement européen, s'aperçoit ensuite que ces personnes venues débattre selon une logique gauche-droite vont toutes se mettre d'accord demain sur l'ensemble des textes qui vont être votés au Parlement européen, et sur la désignation du président du Parlement européen et du président de la Commission. Cela semble davantage incompréhensible chez les Britanniques et les Français parce que la logique majoritaire s'oppose à la logique proportionnelle. Mais je pense voir aussi dans certaines enquêtes portant sur d'autres pays proportionnalistes un décalage entre l'habitude d'un combat gauche-droite et une Europe qui efface ce combat. Sur ce point, il est sûr que nous avons des progrès à faire. Mais les partis politiques aussi ont quelques progrès à faire pour nous expliquer tout cela.

T. Petersen : En Allemagne, on observe une décrispation progressive du débat droite-gauche. Des années 1950 aux années 1990, les deux camps, de poids comparable, étaient en franche confrontation: d'un côté les chrétiens-démocrates et les libéraux, de l'autre les sociaux-démocrates et, plus récemment, Les Verts. Les électeurs s'identifiaient alors très fortement avec leur parti, et perdre une élection était vécu comme une catastrophe. Mais au fil des mutations de la société, l'identification avec les partis politiques s'est affaiblie, la propension des électeurs à changer de camp au gré des élections augmentant dans le même temps. Le rapport des citoyens à l'Etat s'est modifié. Auparavant, ils se voyaient dépendants de l'Etat, attendant de lui directives ou prestations, mais ne s'en sentaient pas partie intégrante. Dans une étude en sciences politiques des années 1950, la société ouest-allemande était alors décrite comme présentant toutes les caractéristiques d'une "subject political culture », ce qui a été fort malencontreusement traduit par "Untertankultur ", c'est-à-dire une culture de sujétion, une formulation totalement inappropriée.

L'approche des Allemands a évolué au fil des décennies. Ils sont de plus en plus nombreux aujourd'hui à penser que leur participation est réelle. Sous le discours sur la désaffection du politique se cache en réalité un engagement politique plus prononcé que jamais, comme le montre l'augmentation de la part de ceux qui affirment que, quand on veut, on peut faire changer les choses. Dans cette nouvelle culture de la participation (la « participative political culture » que décrivent Almond/Verba dans leur ouvrage The civic Culture paru en 1963), le
Logique majoritaire en France et en Grande-Bretagne versus logique proportionnelle

Le PE efface la logique d'affrontement gauche-droite caractéristique de la France

Décrispation du débat gauche-droite en Allemagne

L'Allemagne est aujourd'hui une démocratie mature 
La culture du libéralisme existe en France aussi, dans certaines régions

Les Français sont mal informés

Les Allemands aussi

Les Français doutent de la démocratie

II n'y a pas un problème d'offre, mais de demande d'information citoyen se voit comme acteur. Et dès lors, il ne vit plus comme une catastrophe le fait que le 'mauvais' parti soit au gouvernement, mais considère qu'il est en son pouvoir de changer cela. Certes, la confrontation entre les camps politiques perdure, mais elle a perdu son caractère conflictuel. L'Allemagne est aujourd'hui une démocratie mature.

C. LEQUESNE : Ce que vous dites rejoint finalement la culture du libéralisme, celle qui consiste à penser que la société doit d'abord s'organiser avant d'attendre l'intervention de l'Etat. Sur ce point, il y a peut-être une différence importante avec la France. II m'a frappé de constater que la région Ouest est celle qui vote le moins pour le FN. En effet, dans le Grand Ouest, le FN n'arrive pas en tête. Toutes les études sociologiques nous disent que c'est une région où le tissu social est très structuré, avec de nombreuses associations, une prise en charge du quotidien et une résolution des problèmes par les individus et par la société. II y a peut-être un lien ici - qui est aussi important que la question de la présence ou non des immigrés - dans l'explication de la variable de vote.

\section{Sommes-nous des citoyens éclairés ?}

I. BouRGEOIs : Ne faudrait-il pas aussi ajouter à cette variable une information déficiente sur les questions européennes?

R. CAYRol : Les médias ne jouent pas suffisamment bien leur rôle en France. A la fin de la campagne européenne, toutes les chaînes se sont battues pour ne pas retransmettre le débat entre les prétendants à la présidence de la Commission européenne. Même le service public a refusé de le diffuser. Elles ont accepté de le faire seulement sur Internet, avec l'idée que dès qu'on parle de l'Europe, l'audience fuit. En effet, à chaque fois que des émissions télévisées ont été diffusées autour des objectifs européens et des débats intereuropéens, cela n'a pas bien fonctionné. Les médias sont coupables, mais les citoyens pourraient aussi de temps en temps se montrer plus responsables. Nous avons voté pour des élections européennes et pourtant, dans nos enquêtes, nous n'avons pas rencontré de citoyen européen qui sache ce qu'est le Parlement européen. Nous n'éprouvons même pas le besoin de savoir pourquoi on va voter. II y a là quelque chose qui en dit beaucoup, non pas sur la désaffection par rapport à l'Europe en général, mais par rapport aux institutions européennes. On constate le manque de pédagogie et de volonté d'apprendre à la fois.

T. Petersen : En Allemagne aussi. En 2009, année de l'entrée en vigueur du Traité de Lisbonne qui a apporté des modifications fondamentales au fonctionnement de l'UE, j'ai fait une analyse du contenu de l'information du magazine Der Spiegel, média leader en Allemagne. Seul quelque 0,8\% de toute l'information avait trait à l'Europe. Pas un article dans le premier tiers des numéros, pas un de plus d'une page. Le poids de l'attention médiatique accordée à cette profonde réforme de l'UE était dérisoire. Peu après, l'IfD a demandé aux Allemands : “Savez-vous qui est Hermann Van Rompuy ? ». Seuls $2 \%$ le savaient.

C. LEQUESNE : En 2005, lors du référendum en France, il y a eu un débat public très vivace, pas seulement sur les réseaux sociaux, mais dans toutes les villes. Les Français étaient devenus des 'experts' passionnés du projet de traité constitutionnel. Je pense que le fait que malgré le rejet de ce texte, nous avons ensuite adopté par voie parlementaire à peu près exactement le même traité n'a pas été très bon pour que vive dans ce pays le sentiment que l'Europe était démocratique. Non seulement les $55 \%$ de Français qui ont voté non ont estimé qu'ils avaient été privés de leur victoire, mais également beaucoup d'électeurs qui avaient voté oui ont ressenti un malaise par rapport au fait que, deux à trois ans après, un texte proche était adopté quand même. Cet oubli du peuple pèse sur l'image de la démocratie européenne.

Je ne suis pas de l'avis que c'est la presse qui fait le débat public. Je pense que c'est le débat public qui influence la presse et que si on parle peu de l'Europe 
dans les journaux, c'est tout simplement parce que la demande est assez faible. Quant à l'école, on constate un vrai effort des professeurs d'histoire et de géographie pour enseigner l'Europe en classe de terminale. Pour ce qui est de l'Europe économique par les professeurs de sciences économiques et sociales, il y aurait beaucoup à dire ...

T. Petersen : Les théories de la communication de ces 50 dernières années nous ont appris au contraire que ce sont les médias qui déterminent de quoi on débat dans l'espace public, qui en fixent l'ordre du jour : c'est la fonction de l'agenda setting. Autrement dit, si nous voulons qu'ils prennent au sérieux les questions européennes, qu'ils considèrent que les élections européennes sont là pour élire un Parlement européen et non pour désavouer un gouvernement national, il nous faut convaincre les journalistes de l'importance de l'Europe. Ce n'est qu'ainsi que nous parviendrons à amener les citoyens à s'intéresser à l'Europe.

C. LEQUESNE : Je ne surestimerais pas le pouvoir d'agenda setting de la presse, ceci d'autant plus qu'aujourd'hui, la communication ne passe plus uniquement par les journalistes ; elle passe de plus en plus par d'autres formes d'échanges, en particulier tous les réseaux sociaux. Par ailleurs, il est difficile de vulgariser le thème de l'Europe (et de la mondialisation), notamment à partir de catégories simples que nous connaissons dans la politique. On revient toujours à des grilles de lecture. Or tout cela, quand on parle d'Europe, n'entre pas très souvent dans ces grilles de lecture gauche/droite, majorité/opposition auxquelles les cultures politiques nationales nous ont habitués.

\section{Quelles perspectives pour l'union politique de l'Europe?}

R. CAYrol : Le thème de la démocratie en Europe est important. On nous a annoncé lors de la campagne que désormais, le candidat proposé par le Conseil européen pour la présidence de la Commission devrait être élu par la majorité du Parlement européen. Je sais bien qu'il peut y avoir discussion sur ce que veut dire le «prendre en compte » du Traité de Lisbonne, mais il ne peut pas y avoir de discussion sur le fait qu'il y ait un vote majoritaire du Parlement européen. Je ne suis pas un admirateur de Jean-Claude Juncker, mais je suis de ceux qui pensent que s'il n'était pas président de la Commission européenne alors qu'il est le représentant du parti le plus important, cela ternirait de nouveau l'image de la démocratie en Europe. Je ne donnerais pas cher non plus de l'image du Conseil européen.

C. LEQUESNE : Si le Conseil européen écarte Jean-Claude Juncker, il s'agira d'une régression de la démocratie européenne. Pour l'instant, nous en sommes aux procédures. Or David Cameron, qui se prononce contre parce qu'il est défié chez lui par ceux qui sont favorables à la sortie de la Grande-Bretagne de l'Europe, a quelques soutiens de la part des chefs d'Etat et de gouvernement. Mais Angela Merkel, bien qu'elle n'ait pas une passion particulière pour Jean-Claude Juncker, est prête à le soutenir dans un compromis final.

H. UTERWEDDE : II y a des limites au consensus et il faut aussi demander à tous, David Cameron inclus, d'accepter un vote démocratique et un vote à la majorité. Une partie de la crédibilité de l'Europe et du Parlement est en jeu.

R. CAYRoL : Bien que j'aie essayé d'être plutôt positif dans mon regard sur les élections européennes, sans m'étendre sur leur ambiance incroyablement eurosceptique, mon opinion est que, dans l'état du débat européen, les gouvernants des Vingt-Huit sont devant une nécessité vitale de faire plus d'Europe et d'accepter de véritables transferts de souveraineté. Ce n'est qu'à cette condition qu'elle pourra à nouveau susciter un espoir dans l'opinion et dans la réalité de nos sociétés.

C. LEQUESNE : Je pense qu'il nous faut un nouvel agenda positif qui ne soit pas simplement un agenda de gestion de crise, ce qui est le cas depuis 2008. II va
Et la fonction

d'agenda setting de la presse?

Elle semble mise à mal par les réseaux sociaux

Le respect de la démocratie veut que J.-C. Juncker soit élu président de la Commission

Accepter les transferts de souveraineté

II faut un nouvel agenda positif 
Régler d'abord les problèmes au niveau national

Intégrer davantage la notion de subsidiarité falloir trouver de nouvelles politiques porteuses. Autour de l'énergie, quelque chose se dessine qui, à défaut de faire consensus, comme le montre l'exemple franco-allemand, peut tout de même constituer un thème de débat. La controverse a toujours quelque chose de positif.

H. UTERWEDDE : Je pense qu'il est possible de vaincre l'euroscepticisme. Quand la situation économique d'un Etat est favorable, la tentation est certes peut-être moins grande d'accuser l'Europe libérale et l'austérité. Or il faut arrêter de se voiler la face. L'Europe n'est pas la cause des problèmes actuels de la France. La France pourrait mettre en œuvre en toute autonomie une réforme administrative ou une réforme de la formation professionnelle, face au chômage des jeunes qui sévit depuis des décennies et à la difficulté de passer de la vie scolaire à la vie professionnelle. L'Europe n'empêche pas non plus la France de faire une réforme des universités, d'instaurer une fiscalité et une politique de contributions favorables à l'emploi et à la compétitivité et de mettre en place un cadre réglementaire propice aux entreprises. II faudrait donc commencer par régler les problèmes au niveau national. Et il faut faire la part de ce qui est imputable à l'Europe et de ce que peut faire l'Europe. On peut espérer un nouvel agenda européen, dans une réflexion commune. Toutefois, je ne crois pas trop à un agenda doté de nombreux milliards pour remédier au chômage des jeunes. L'Europe, c'est un grand marché qui doit être organisé et qui est propice au développement et à la croissance s'il fonctionne vraiment.

POUR CONCLURE, NOUS N'ECHAPPERONS PAS A UN DEBAT DE FOND sur l'avenir de l'Europe. Que sommes-nous prêts à faire ensemble, à partager en termes de compétences et de ressources ? Après l'élection du Parlement et au regard des évènements autour de l'énergie et de la Russie notamment, le moment est peut-être venu de mener un grand débat collectif européen sur ces questions. Le statu quo mènera dans le mur. II nous faut choisir entre la régression d'un côté et, de l'autre, une marche réfléchie vers toujours plus d'Europe, en établissant ce qu'on devra faire en commun en Europe et ce qui peut rester au niveau national. C'est là qu'intervient le maître-mot de «subsidiarité ». Cette notion a été très présente dans le débat européen allemand. Elle signifie faire faire à l'Europe ce qui est vraiment essentiel là où l'Europe est mieux placée que les Etats et les régions pour en assumer les charges, mais aussi laisser aux Etats et aux régions le soin de gérer tout le reste. Or tout le reste, c'est beaucoup. Par conséquent, plus d'Europe peut signifier aussi plus de concentration de l'Europe sur les vraies tâches communes et peut-être moins d'Europe dans d'autres tâches secondaires. II s'agit d'un vaste agenda non seulement pour ceux qui nous gouvernent, mais aussi pour les citoyens. II faudra essayer d'organiser et de nourrir ces débats sur l'avenir de l'Europe dans nos pays, mais aussi à travers des cercles franco-allemands et européens.

Synthèse du débat : Isabelle Bourgeois, avec le concours de Solène Hazouard et de Mélanie Prieur

Ce débat s'est tenu le 2 juin à la Maison Heinrich Heine à Paris. Il était organisé par le CIRAC, en coopération avec le Centre de recherche Civilisations et identités culturelles comparées (CICC) de l'Université de Cergy-Pontoise (UCP), le DFI, le Centre interdisciplinaire d'études et de recherches sur l'Allemagne (CIERA), et avec le concours de la Fondation de l'UCP.

Ce débat constituait le troisième volet du cycle thématique «France-Allemagne-Europe » proposé en 2014 par l'Institut d'études avancées de l'Université de Cergy-Pontoise (IEA). Il fait suite à deux conférences-débats :

- la première s'était tenue le 13 mars 2014 à l'Université de Cergy-Pontoise, sur le thème «La nouvelle constellation politique franco-allemande : un nouveau souffle pour l'Europe ? » ;

- la deuxième, organisée le 29 avril 2014 au Goethe-Institut de Paris, était intitulée : « Les positions française et allemande sont-elles conciliables face aux enjeux de l'union politique et de la gouvernance économique européennes? ».

En savoir plus : www.cirac.u-cergy.fr. 